\title{
ON THE CORRECTNESS OF PARTIAL DIFFERENTIAL OPERATORS AND THE VON NEUMANN CONDITION FOR STABILITY OF FINITE DIFFERENCE OPERATORS ${ }^{1}$
}

\author{
D. G. ARONSON
}

1. Introduction. Let $P(\xi)$ be an $N \times N$ matrix whose elements are polynomials of degree $\leqq m$ in $\xi=\left(\xi_{1}, \cdots, \xi_{n}\right)$ and let

$$
L \equiv P(D)-E \partial / \partial t
$$

where $D=\left(\partial / \partial x_{1}, \cdots, \partial / \partial x_{n}\right)$ and $E$ is the $N \times N$ identity matrix. The operator $L$ is said to be correct in the sense of Petrovskit [3] if there exists a constant $K>0$ such that

$$
\max _{,} \operatorname{Re} \mu_{j}(\xi) \leqq K \quad \text { for all } \xi \in E^{n}
$$

where the $\mu_{j}(j=1,2, \cdots, N)$ are the eigenvalues of $P(i \xi)$. We shall give a necessary and sufficient condition for the correctness of $L$ in terms of finite difference approximations to $L$.

Let $h$ and $\tau$ be arbitrary positive numbers, and let

$$
R_{h, \tau}=\left\{(j h, l \tau) \mid j \in I^{n}, \quad l=0,1, \cdots\right\},
$$

where $I^{n}$ is the set of all $n$-tuples of integers. As approximations to $L$ we shall consider the two-parameter family of two-level finite difference operators

$$
L_{h, \tau} u(x, t) \equiv \sum_{r} B_{r}^{(0)} u(x+r h, t)+\sum_{r} B_{r}^{(1)} u(x+r h, t+\tau)
$$

for $(x, t) \in R_{h, r}$, where the $B_{r}^{(i)}$ are $N \times N$ matrices which depend only on $h$ and $\tau, r \in I^{n}$, and $B_{r}^{(i)}=0$ outside some fixed finite neighborhood of $r=0$. The family of finite difference operators $\left\{L_{h, \tau}\right\}$ is said to be consistent with $L$ if

$$
\left(L_{h, \tau}-L\right) u(x, t) \rightarrow 0 \quad \text { as } h, \tau \rightarrow 0
$$

for all sufficiently smooth $u$ [7].

For any $\alpha \in E^{n}, L_{h, \tau} u=0$ has a solution of the form $u(x, t)=e^{i \alpha^{T}}{ }^{x} q(t)$ if and only if $q$ satisfies

Received by the editors July 16, 1962.

1 This work was supported in part by the Office of Naval Research and in part by the National Science Foundation under a Postdoctoral Fellowship. 


$$
\left(\sum_{r} B_{r}^{(0)} e^{i \alpha_{r h}}\right) q(t)+\left(\sum_{r} B_{r}^{(1)} e^{i \alpha^{T} r h}\right) q(t+\tau)=0
$$

for $t=l \tau$. Let

$$
H_{j}(h, \tau, \alpha)=\sum_{r} B_{r}^{(j)} e^{i \alpha^{T} \boldsymbol{r}} \quad(j=0,1) .
$$

The family of finite difference operators $\left\{L_{h, \tau}\right\}$ is said to satisfy the von Neumann condition for stability if there exists a positive rational number $a$, and positive constants $h_{0}$ and $\lambda_{0}$ such that for all $\alpha \in E^{n}$ and $h \leqq h_{0}$, and for every subfamily $\left\{L_{h, \lambda}\right\}$ of $\left\{L_{h, \tau}\right\}$ with $\lambda \equiv \tau h^{-a}$ $=$ constant $\leqq \lambda_{0}$ the eigenvalues $\gamma_{j}=\gamma_{j}(\lambda, h, a, \alpha)$ of $-H_{1}^{-1} H_{0}$ all satisfy

$$
\left|\gamma_{j}\right| \leqq 1+c \tau,
$$

where $c$ is a positive constant which may depend on $a, h_{0}$ and $\lambda$. The von Neumann condition is necessary for the stability of solutions of the initial value problem for $\left\{L_{h, \tau}\right\}$ (see [7] and $\$ 3$ below).

In $\$ 2$ we shall prove

Theorem 1. The differential operator $L$ is correct in the sense of Petrovskiz if and only if there exists at least one family of two-level finite difference operators $\left\{L_{h, \tau}\right\}$ which is consistent with $L$ and which satisfies the von Neumann condition for stability.

In view of the connection between correctness in the sense of PetrovskiY and the von Neumann condition for stability it is reasonable to expect to be able to prove finite difference analogues of known results concerning the initial value problem with correct $L$ for the initial value problem for a family $\left\{L_{h, r}\right\}$ which satisfies the von Neumann condition. We consider this question in $\S 3$, where we show that for explicit two-level finite difference operators the von Neumann condition is sufficient for stability in the sense of Rjaben'kiY and Filippov [8].

2. Proof of Theorem 1. Assume that $L$ is correct in the sense of Petrovski1, i.e., that (1.1) holds. Let $\delta=\left(\delta_{1}, \cdots, \delta_{n}\right)$, where $\delta_{j} u(x, t)$ $=u\left(x+e_{j} h, t\right)-u\left(x-e_{j} h, t\right)$ and $e_{j}$ is the $j$ th unit coordinate vector in $E^{n}$, and let $\Delta u(x, t)=u(x, t+\tau)-u(x, t)$. The family of two-level finite difference operators

$$
L_{h, \tau} \equiv P(\delta / 2 h)-\tau^{-1} E \Delta
$$

is clearly consistent with $L$. We claim that $\left\{L_{h, \tau}\right\}$ satisfies the von 
Neumann condition. In this case $H_{1}=-\tau^{-1} E$ and $H_{0}=\tau^{-1} E$ $+P(i \sin \alpha h / h)$, where $\sin \alpha h=\left(\sin \alpha_{1} h, \cdots, \sin \alpha_{n} h\right)$. Thus $-H_{1}^{-1} H_{0}$ $=E+\tau P(i \sin \alpha h / h)$ and it follows that

$$
\left|\gamma_{j}\right|^{2}=1+\tau\left\{2 \operatorname{Re} \mu_{j}(\sin \alpha h / h)+\tau\left|\mu_{j}(\sin \alpha h / h)\right|^{2}\right\} .
$$

By a theorem of Gel'fand and Silov [3, p. 83], there exists a constant $c_{1}>0$ such that

$$
\max _{1}\left|\mu_{j}(\xi)\right| \leqq c_{1}(1+|\xi|)^{m_{0}}
$$

where $|\xi|^{2}=\sum_{k=1}^{n} \xi_{k}^{2}, m_{0}=\max _{1 \leqq k \leq N}\left(p_{k} / k\right)$, and $p_{k}$ is the degree of the coefficient of $\mu^{N-k}$ in the characteristic polynomial of $P(i \xi)$. If we use (1.1), (2.3) and the fact that $|\sin \alpha h| \leqq n^{1 / 2}$ in (2.2) we obtain

$$
\left|\gamma_{j}\right|^{2} \leqq 1+\tau\left\{2 K+c_{1}^{2} \tau h^{-2 m_{0}}\left(h+n^{1 / 2}\right)^{2 m_{0}}\right\} \text {. }
$$

Thus if we set $\lambda=\tau h^{-2 m_{0}}$ and $h_{0}=1$, the von Neumann condition holds for arbitrary $\lambda_{0}>0$.

Now assume that we have a family of finite difference operators $\left\{L_{h, \tau}\right\}$ which is consistent with $L$ and for which the von Neumann condition holds. Choose any $\lambda \leqq \lambda_{0}$ and consider the subfamily $\left\{L_{h, \lambda}\right\}$. Write $-H_{1}^{-1} H_{0}$ in the form

$$
-H_{1}^{-1} H_{0}=E+\tau P_{h, \lambda}(\alpha) \text {. }
$$

We assert that for each fixed $\alpha \in E^{n}$

$$
\lim _{h \rightarrow 0} P_{h, \lambda}(\alpha)=P(i \alpha) \text {. }
$$

Let $u_{1}(x)=e^{i \alpha^{T} x} E$ and $u_{2}(x, t)=t u_{1}(x)$. If we apply (1.2) first to $u_{1}$ and then to $u_{2}$ we obtain $-\left(H_{0}+H_{1}\right)=P(i \alpha)+A_{1}$ and $\tau H_{1}=E+A_{2}$, where $A_{j} \rightarrow 0$ as $h \rightarrow 0$ for each fixed $\alpha \in E^{n}$. In particular, it follows that $\left(\tau H_{1}\right)^{-1}=E+A_{3}$, where $A_{3} \rightarrow 0$ as $h \rightarrow 0$ for fixed $\alpha$. Thus $-\tau^{-1}\left(E+H_{1}^{-1} H_{0}\right)=P(i \alpha)+A_{3} P(i \alpha)+A_{1}+A_{3} A_{1}$, which proves the assertion.

Let $\mu_{j}^{(h, \lambda)}(\alpha)$ denote the eigenvalues of $P_{h, \lambda}(\alpha)$. In view of (2.4) we have $\gamma_{j}=1+\tau \mu_{j}^{(h, \lambda)}$. Since the von Neumann condition holds, it follows from (1.3) that

$$
\left|\gamma_{j}\right|^{2}=1+\tau\left(2 \operatorname{Re} \mu_{j}^{(h, \lambda)}+\tau\left|\mu_{j}^{(h, \lambda)}\right|^{2}\right) \leqq 1+\tau c\left(2+h_{0} \lambda(c)\right.
$$

for all $h \leqq h_{0}$ and $\alpha \in E^{n}$, where $c=c\left(a, h_{0}, \lambda\right)$. In particular,

$$
\operatorname{Re} \mu_{j}^{(h, \lambda)}(\alpha) \leqq c\left(1+\frac{1}{2} h_{0}^{a} \lambda c\right) \equiv \tilde{c}\left(a, h_{0}, \lambda\right)
$$


for all $h \leqq h_{0}$ and $\alpha \in E^{n}$. On the other hand, (2.5) implies that $\mu_{j}^{(h, \lambda)}(\alpha) \rightarrow \mu_{j}(\alpha)$ as $h \rightarrow 0$ for each fixed $\alpha \in E^{n}$. Thus it follows from (2.6) that $\operatorname{Re} \mu_{j}(\alpha) \leqq \tilde{c}\left(a, h_{0}, \lambda\right)$ for all $\alpha \in E^{n}$, i.e., $L$ is correct in the sense of Petrovskir.

REMARK. Although, as we have proved, $\tau h^{-2 m_{0}}=$ constant implies the von Neumann condition for the difference operators (2.1), in certain special cases an exponent smaller than $2 m_{0}$ will suffice. For example, if $L$ is parabolic in the sense of Petrovskil [3], i.e., if $\operatorname{Re} \mu_{j}(\xi) \leqq-\rho|\xi|^{m}$ for some $\rho>0$, then $m_{0}=m$ but the von Neumann condition holds for (2.1) if $\tau h^{-m}$ is suitably restricted [2]. On the other hand, if for $n=1$

$$
P(i \xi)=\left(\begin{array}{cc}
0 & i \xi \\
i \xi & 0
\end{array}\right)
$$

(i.e., $L u=0$ is the one-dimensional wave equation) then $m_{0}=1$ and $\tau h^{-2}=$ constant is also necessary for the von Neumann condition. In this case, and in general, the situation can be improved by using a more sophisticated difference approximation in place of (2.1) (see $[7$, p. 168]).

3. Stability. We now restrict our attention to the class of explicit finite difference operators

$$
\mathscr{L}_{h, r} u(x, t) \equiv \sum_{r} B_{r} u(x+r h, t)-\tau^{-1} \Delta u(x, t) .
$$

For example, the difference operators (2.1) are clearly explicit. Moreover, the two-level approximation to the one-dimensional wave operator discussed in $[7$, formula $(8.5)$, p. 168] is also explicit since it can be written in the form

$$
\mathfrak{L}_{h, \tau}=\left(\begin{array}{cc}
0 & \frac{c}{2 h} \delta \\
\frac{c}{2 h} \delta & \frac{c^{2} \tau}{4 h^{2}} \delta^{2}
\end{array}\right)-\frac{1}{\tau}\left(\begin{array}{cc}
\Delta & 0 \\
0 & \Delta
\end{array}\right) .
$$

We shall assume that $\left\{\mathfrak{L}_{h, \tau}\right\}$ is consistent with a fixed differential operator $L \equiv P(D)-E \partial / \partial t$ and that the von Neumann condition holds for $\left\{\mathscr{L}_{h, \tau}\right\}$. (Thus $L$ is correct.) Let $g=g(x)$ be a given $N$-vector defined on $E^{n}$. We consider the two-parameter family of initial value problems

$$
\begin{aligned}
\mathscr{L}_{h, \tau} u(x, t) & =0 \quad \text { for }(x, t) \in R_{h, \tau}(T) ; \\
u(j h, 0) & =g(j h) \quad \text { for } j \in I^{n},
\end{aligned}
$$


where

$$
R_{h, \tau}(T)=\left\{(j h, l \tau) \mid j \in I^{n}, l=0,1, \cdots, T / \tau-1\right\}
$$

for arbitrary $T>0$ and $\tau$ chosen so that $T / \tau=$ integer. Since $\mathscr{L}_{h, \tau}$ is explicit and $r$ ranges over a finite subset of $I^{n}$, it is clear that the initial value problem has a unique solution for every positive $h$ and $\tau$.

Let $\lambda$ be a positive constant and consider the one-parameter subfamily $\left\{\mathscr{L}_{h, \lambda}\right\} \subset\left\{\mathfrak{L}_{h, \tau}\right\}$ characterized by $\tau h^{-a}=\lambda$ for some fixed positive rational number $a$. The one-parameter family of initial value problems (3.2) corresponding to $\left\{\mathfrak{L}_{h, \lambda}\right\}$ is said to be stable if it is uniformly well-posed for all sufficiently small $h$, i.e., if there exist norms $\|\cdot\|_{0}$ and $\|\cdot\|_{1}$, and positive constants $h_{0}$ and $\sigma=\sigma\left(a, h_{0}, \lambda\right)$, such that for every $h \leqq h_{0}$ the solution $u=u_{h, \lambda}(x, t)$ satisfies $\|u\|_{1} \leqq \sigma\|g\|_{0}$. The problem of stability of (3.2) has been studied by various authors. In the work of Lax and Richtmyer [7] and Kreiss $[5 ; 6],\|g\|_{0}$ is the $L_{2}\left(E^{n}\right)$-norm and $\|u\|_{1}=\sup _{[0, T]}\left(\int_{E^{n}}|u|^{2} d x\right)^{1 / 2}$. Kreiss [5] gives a necessary and sufficient condition for stability in this sense. John [4] and Aronson $[1 ; 2]$ use the uniform norm for $\|g\|_{0}$ and a norm involving the first $m-1$ differences of $u$ for $\|u\|_{1}$. In all of these works the von Neumann condition is necessary but, in general, not sufficient for stability. In this paper, as in the work of Rjaben'kiY and Filippov [8], $\|u\|_{1}$ will be the uniform norm and $\|g\|_{0}$ will involve various derivatives (or differences) of $g$. We shall show that the von Neumann condition is sufficient for stability in this sense.

For explicit difference operator $\mathfrak{L}_{h, \tau}$ we have $-H_{1}^{-1} H_{0}=E+\tau P_{h, \tau}(\alpha)$, where

$$
P_{h, \tau}(\alpha)=\sum_{r} B_{r} e^{i \alpha^{T} r h}
$$

Since $\left\{\mathscr{L}_{h, \tau}\right\}$ is consistent with $L$, we have

$$
P_{h, \tau}(\alpha) \rightarrow P(i \alpha) \quad \text { as } h, \tau \rightarrow 0
$$

for each fixed $\alpha \in E^{n}$. Write $P(\xi)=\sum_{l=0}^{m} P_{l}(\xi)$, where the elements of $P_{l}(\xi)$ are homogeneous polynomials of degree $l$ in $\xi$. It follows from (3.3) and (3.4) that

$$
\frac{(i h)^{l}}{l !} \sum_{r} B_{r}\left(\alpha ^ { T _ { r } ) ^ { l } } \rightarrow \left\{\begin{array}{cl}
P_{l}(i \alpha) & \text { for } l=0,1, \cdots, m, \\
0 & \text { for } l=m+1, m+2, \ldots
\end{array}\right.\right.
$$

as $h, \tau \rightarrow 0$ for fixed $\alpha \in E^{n}$. The matrices $B_{r}=0$ for $r$ outside a finite neighborhood of $r=0$. Let $r^{(1)}, \cdots, r^{(s)}$ be the set of all $r \in I^{n}$ such that $r \neq 0$ and $B_{r} \neq 0$. Using Cramer's rule, it is not difficult to verify 
from (3.5) that $h^{\bullet} B_{r}$ is bounded as $h, \tau \rightarrow 0$ for all $r$. Let $q$ be the smallest integer for which $h^{a} B_{r}$ is bounded as $h, \tau \rightarrow 0$ for all $r$. Clearly $q \leqq s$. It is possible to have $q<s$. For the operator (2.1) we have $s=2 m$ and $q=m$, and for the operator (3.1) we have $s=4$ and $q=2$. In general, if $\mathfrak{L}_{h, \tau}$ involves only centered $x$-differences $\delta$, then $q=s / 2$. It follows from Taylor's theorem and (3.3) that ${ }^{2}$

$$
\left|P_{h, \tau}(\alpha)\right| \leqq \sum_{l=0}^{q-1}|\alpha|^{l}\left|\frac{(i h)^{l}}{l !} \sum_{r} B^{r}\left(\frac{\alpha^{T} r}{|\alpha|}\right)^{l}\right|+\frac{|\alpha|^{q}}{q !} \sum_{r} h^{q}\left|B_{r}\right||r|^{q} .
$$

Thus in view of (3.5) and the definition of $q$, there exist positive constants $h_{0}, \tau_{0}$, and $c_{1}=c_{1}\left(h_{0}, \tau_{0}, q, s\right)$ such that

$$
\left|P_{h, r}(\alpha)\right| \leqq c_{1}(1+|\alpha|)^{q} \text { for all } h \leqq h_{0}, \tau \leqq \tau_{0} \text { and } \alpha \in E^{n} .
$$

Let $a_{\nu}$, where $\nu$ is a non-negative integer, denote the class of all $N$-vectors $g=g(x)$ defined on $E^{n}$ which are such that $g \in C^{v}\left(E^{n}\right)$ and $D^{k} g \in L\left(E^{n}\right)$ for $|k| \leqq \nu$. If $g \in Q_{\nu}$ we shall write

$$
\|g\|_{\nu}=\max _{|k| \leq \nu}\left(\int_{E^{n}}\left|D^{k} g\right| d x\right) \text {. }
$$

Let

$$
\bar{R}_{h, \tau}(T)=\left\{(j h, l \tau) \mid j \in I^{n}, l=0,1, \cdots, T / \tau\right\} .
$$

For $u=u(x, t)$ defined on $\bar{R}_{h, \tau}(T)$ we shall write

$$
\|u\|=\max _{\bar{R}_{h, \tau}(T)}|u(x, t)| \text {. }
$$

We shall prove

THEOREM 2. Let the family $\left\{\mathfrak{L}_{h, \tau}\right\}$ of explicit finite difference operators be consistent with a fixed differential operator $L$ and satisfy the von Neumann condition. For any $\lambda \leqq \lambda_{0}$ let $u=u_{h, \lambda}(x, t)$ denote the solution of (3.2) for $\mathfrak{L}_{h, \lambda}$. Then if $g \in Q_{\nu}$, for $\nu=q(N-1)+n+1$, there exists a constant $\sigma=\sigma\left(a, h_{0}, \lambda, q, s, N, T\right)$ such that

$$
\|u\| \leqq \sigma\|g\|, \quad \text { for all } h \leqq h_{0} .
$$

Proof. Let $\hat{g}(\alpha)$ denote the Fourier transform of $g(x)$. Since $g \in a_{\text {, }}$ it is easy to verify that $|\hat{g}(\alpha)| \leqq(n+1)^{\nu}(1+|\alpha|)^{-v}\|g\|_{\nu}$. Assume that there is a constant $c_{2}>0$ such that

$$
\left|\left\{E+\tau P_{h, \lambda}(\alpha)\right\}^{\imath}\right| \leqq c_{2}(1+|\alpha|)^{g(N-1)}
$$

${ }^{2}$ If $u=\left(u_{1}, \cdots, u_{N}\right)$ then $|u|=\sum_{f_{1}}^{N}\left|u_{j}\right|$. If $A=\left(a_{i j}\right)$ is an $N \times N$ matrix then $|A|=N \max _{i, j}\left|a_{i j}\right|$. Note that $|A u| \leqq|A||u|$. 
for all $h \leqq h_{0}, \alpha \in E^{n}$ and $l \tau \leqq T$. Let

$$
u(x, l \tau)=(2 \pi)^{-n / 2} \int_{E^{n}}\left\{E+\tau P_{h, \lambda}(\alpha)\right\}^{l} g(\alpha) e^{i \alpha^{T} x} d \alpha .
$$

Then

$$
|u(x, l \tau)| \leqq(2 \pi)^{-n / 2}(n+1)^{\nu} c_{2}\|g\|_{\nu} \int_{E^{n}}(1+|\alpha|)^{-(n+1)} d \alpha,
$$

and $u(j h, l \tau)$ is clearly the solution of (3.2). Thus we must show that (3.7) holds.

By a theorem of Schur (quoted in [7, p. 67]) there exists a unitary matrix $U=U_{h, \lambda}(\alpha)$ such that $U^{*} P_{h, \lambda}(\alpha) U=\tilde{P}_{h, \lambda}(\alpha)=\left(\tilde{p}_{i j}^{(h, \lambda)}(\alpha)\right)$, where $\tilde{p}_{i i}^{(h, \lambda)}=\mu_{i}^{(h, \lambda)}, \tilde{p}_{i j}^{(h, \lambda)}=0$ for $i>j$, and $\left|\tilde{p}_{i j}^{(h, \lambda)}\right| \leqq N^{2} \max _{k, l}\left|p_{k l}^{(h, \lambda)}\right|$. Thus $\left\{E+\tau P_{h, \lambda}(\alpha)\right\}^{l}=U\left\{E+\tau \widetilde{P}_{h, \lambda}(\alpha)\right\}^{l} U^{*}$. Write $E+\tau \widetilde{P}_{h, \lambda}=\Gamma+\tau \bar{P}_{h, \lambda}$, where $\Gamma$ is the diagonal matrix with elements $\gamma_{j}=1+\tau \mu_{j}^{(h, \lambda)}$. In view of (3.6), $\left|\bar{P}_{h, \lambda}\right| \leqq c_{1} N^{3}(1+|\alpha|)^{q}$. Moreover, according to (1.3), $\left|\gamma_{j}\right|$ $\leqq 1+c \tau$. Thus for $l>N-1$, taking account of the structure of $\Gamma$ and $\bar{P}_{h, \lambda}$, we find

$$
\left|\left(E+\tau \tilde{P}_{h, \lambda}\right)^{l}\right| \leqq \sum_{j=0}^{N-1}(N \tau)^{j} C_{l, j}(1+c \tau)^{l-j}\left\{c_{1} N^{3}(1+|\alpha|)^{q}\right\}^{j} \equiv M .
$$

Since $C_{l, j} \leqq l^{j} / j$ ! and $l \tau \leqq T$ we obtain

$$
M \leqq N^{N-1}(1+|\alpha|)^{q(N-1)} e^{c T} \sum_{j=0}^{N-1}\left(c_{1} T N^{3}\right)^{j} / j ! \equiv c_{3}(1+|\alpha|)^{q(N-1)},
$$

where $c_{3}=c_{3}\left(a, h_{0}, \lambda, q, s, N, T\right)$. Therefore $\left|\left(E+\tau P_{h, \lambda}\right) l\right|$ $\leqq c_{3} N^{2}(1+|\alpha|)^{q(N-1)}$ for $l \geqq N$. On the other hand, it follows from (3.6) that for $l \leqq N-1,\left|\left(E+\tau P_{h, \lambda}\right)^{l}\right| \leqq\left(N+h_{0}^{a} c_{1} \lambda\right)^{N-1}(1+|\alpha|)^{q(N-1)}$ $\equiv c_{4}(1+|\alpha|)^{q(N-1)}$, where $c_{4}=c_{4}\left(a, h_{0}, \lambda, q, s, N\right)$. Therefore (3.7) holds with $c_{2}=\max \left(c_{3} N^{2}, c_{4}\right)$ and the proof of the theorem is complete.

REMARKs. As Rjaben'kiY and Filippov [8] point out, (3.7) is the finite difference analogue of PetrovskiY's "Bedingung A." Thus we may paraphrase Theorem 2 as follows: The von Neumann condition implies "Bedingung A" which in turn implies stability in the indicated sense. If, in addition to the correctness implied by the hypothesis of Theorem 2, we make further assumptions about the structure of $L$ we can, of course, obtain much stronger results (see, e.g., $[1 ; 2]$ for results concerning parabolic equations and systems). On the other hand, Theorem 2 can be generalized for data of polynomial growth. This, however, would require the deep methods of Gel'fand and Silov $[3]$ and is beyond the scope of this note. 
Stability considerations are important for two reasons. First because, in general, they yield information about the effects of rounding errors on the numerical solution of (3.2), and second because in the appropriate setting, as was shown by Lax [7], stability is necessary and sufficient for the convergence of solutions of (3.2) to the solution of the corresponding initial value problem for $L$. Theorem 2 is not appropriate for the former application. To obtain a better result for this purpose, we assume that $g$ has compact support and let

$$
\|g\|_{h, \nu}=\max _{x \in E^{n},|\boldsymbol{k}| \leq \nu}\left|h^{-|k|} \Delta^{k} g(x)\right|,
$$

where $\Delta=\left(\Delta_{1}, \cdots, \Delta_{n}\right)$ and $\Delta_{j} g(x)=g\left(x+e_{j} h\right)-g(x)$. For such $g$, Rjaben'kiǐ and Filippov [8] have shown that (3.7) implies that $\|u\| \leqq \sigma\|g\|_{h, \nu}$, where $\sigma$ depends on the support of $g$. Since we have shown that the von Neumann condition implies (3.7) it follows that under the hypothesis on $L$ in Theorem $2,\|u\| \leqq \sigma\|g\|_{h, \nu}$. Thus, in particular, if the values of $g$ are known only to within $\pm \epsilon$, then the error from this source in $u$ is bounded by $2^{v} \sigma \epsilon h^{-\nu}$.

\section{REFERENCES}

1. D. G. Aronson, The stability of finite difference approximations to second order linear parabolic differential equations, Duke Math. J. 30 (1963), 117-128.

2. - On the stability of certain finite difference approximations to parabolic systems of differential equations, Numer. Math. 5 (1963), 118-137, 290.

3. I. M. Gel'fand and G. E. Šilov, Generalized functions, Part 3, Gosudarstv. Izdat. Fiz.-Mat. Lit., Moscow, 1958. (Russian)

4. Fritz John, On integration of parabolic equations by difference methods, Comm. Pure Appl. Math. 5 (1952), 155-211.

5. Heinz-Otto Kreiss, Über die Approximative Lösung von linearen partiellen Differentialgleichungen mit Hilfe von Differenzengleichungen, Kungl. Tekn. Högsk. Handl. Stockholm 128 (1958), 1-19.

6. - - Über die Lösung des Cauchyproblems für lineare partielle Differentialgleichungen mit Hilfe von Differenzengleichungen. 1, Acta Math. 101 (1959), 179-199.

7. R. D. Richtmyer, Difference methods for initial-value problems, Interscience Tracts in Pure and Applied Mathematics. Tract 4. Interscience, New York, 1957.

8. V. S. Rjaben'kif and A. F. Filippov, On stability of difference equations, GITTL, Moscow, 1956 (Russian); German transl., Über die Stabilitat von Differenzengleichungen, Berlin, 1960.

STANFORD UNIVERSITY AND University OF MinNESOTA 